\title{
Structural characterization and expression of new gene homologs of GDP-D-mannose-3', 5'-epimerase GME1 in wild and cultivated tomato species
}

\author{
Tyapkina D.Y.*, Slugina M.A. \\ Federal Research Centre "Fundamentals of Biotechnology" RAS, Moscow, Russia \\ * email: daria_t@list.ru
}

GDP-D-mannose epimerase (GME) is considered to be the central enzyme connecting the ascorbate biosynthesis pathway to primary cell wall metabolism. GME1 plays an important role in ascorbate accumulation in leaves and fruits and also is involved in the cell wall and glycoproteins biosynthesis during growth of vegetative organs. In this work, GME1 homologous genes were identified and characterized in 10 cultivated and wild tomato species and the GME1 expression analysis during fruit development and ascorbate accumulation was carried out.

All identified tomato GME1 homologs included six exons; gene length varied from 1962 bp (S. galapagense) to 1985 bp ( $S$. peruvianum), due to InDels in exon sequences. Comparison with the reference genome revealed 136 SNPs and 22 InDels in GME1 homologs Moreover, 134 SNPs were found in green-fruited tomato species and only 5 SNPs in red-fruited species. Five SNPs (T294C, T738A, T783C, T1746A, and C1967T) and 1259ins9 InDel were found to correlated with the genotype of crosspollinated tomato species.

Analysis of the GME1 amino acid sequences revealed only two substitutions, G2E and E281D, which were present only in green-fruited species, but they did not correlate with the levels of ascorbate accumulation in fruits.

GME1 expression analysis in various plant organs of red-fruited and green-fruited accessions revealed the highest transcription levels in the leaves and the lowest in the roots. Comparative analysis between ascorbate content and expression levels in mature fruits in ten S. lycopersicum cultivars and two green-fruited tomato species contrasting in ascorbate content showed no correlation. A comparative analysis of four fruit development stages, ascorbate content and GME1 expression levels showed the maximum number of transcripts in young fruits with a gradual decrease in the expression level to the stage of biological ripeness; this dynamics was opposite to ascorbate accumulation. It is possible that in an intensively growing young fruit, the GME1 enzyme is more actively involved in the process of cell growth rather than in the ascorbate biosynthesis. 